\title{
EEG ultradian rhythmicity differences in disorders of consciousness during wakefulness
}

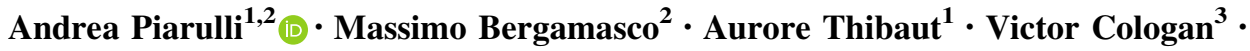 \\ Olivia Gosseries $^{1,4} \cdot$ Steven Laureys ${ }^{1}$
}

Received: 11 January 2016/Revised: 3 June 2016/ Accepted: 4 June 2016

(c) Springer-Verlag Berlin Heidelberg 2016

\begin{abstract}
Temporal fluctuations of cognitively-mediated behaviors in minimally conscious state (MCS) have been linked to changes of awareness, but the time-pattern of these variations remains ill-described. We analyzed 4-h EEG recordings from 12 patients with disorders of consciousness (6 MCS and 6 vegetative state/unresponsive wakefulness syndrome, VS/UWS). Relative powers (delta, theta, alpha, beta1 and beta2 bands) and spectral entropy were estimated (Fz, $\mathrm{Cz}$ and $\mathrm{Pz}$ derivations). Spectral entropy time-courses were then analyzed. MCS patients had higher theta and alpha and lower delta power when compared to VS/UWS. They showed higher spectral entropy mean value and higher time variability. MCS patients were characterized by spectral entropy fluctuations with periodicities of $70 \mathrm{~min}$ (range $57-80 \mathrm{~min}$ ). Notably, these periodicities closely resemble those described in
\end{abstract}

O. Gosseries and S. Laureys contributed equally to this work.

Electronic supplementary material The online version of this article (doi:10.1007/s00415-016-8196-y) contains supplementary material, which is available to authorized users.

Andrea Piarulli

a.piarulli@sssup.it

1 Coma Science Group, GIGA-Research B34, University and University Hospital of Liége, Avenue de l'Hôpital 1, B-4000 Liége, Belgium

2 Perceptual Robotics Laboratory, TECIP Institute, Scuola Superiore Sant'Anna, Via Alamanni 13D, 56010 Pisa, Italy

3 Innovation and Research Management, University Hospital of Toulouse (Hôtel-Dieu Saint-Jacques), 31059 Toulouse, France

4 Department of Psychiatry and Psychology, University of Wisconsin, Madison, WI 53706, USA awake healthy subjects, which were hypothesized to be related to fluctuation in vigilance/attention. No significant periodicity was observed for VS/UWS. The spectral entropy periodicity found in MCS patients could reflect the fluctuation of awareness responsible for the inconsistency of MCS manifestation of cognitively-mediated behaviors. The presence of a 70 min periodicity in spectral entropy could permit clinicians to better choose their time-window when performing a clinical assessment of consciousness. It could also permit to monitor fluctuations in cognitive performance (i.e., response to command) during complementary testing by passive or active electrophysiological or functional neuroimaging paradigms or in resting state conditions.

Keywords Disorders of consciousness, DOC · EEG spectral entropy - Awareness temporal fluctuations . Minimally conscious state, MCS - Vegetative state/ unresponsive wakefulness syndrome, VS/UWS

\section{Introduction}

Clinicians and scientists have been investigating ways to better characterize residual brain function and more accurately diagnose disorders of consciousness. Among those, patients with vegetative state/unresponsive wakefulness syndrome, VS/UWS, [1], retain eyes-opening but demonstrate no behavioral signs of awareness. In contrast, patients in minimally conscious state, MCS, [2], are characterized by fluctuating and inconsistent but reproducible cognitively-mediated behaviors, i.e., non-reflex behavior (MCS minus) or response to command (MCS plus, [3]). While these inconsistencies have been often linked to temporal fluctuations of vigilance/awareness [4-6], to the 
best of our knowledge, no one has investigated the pattern over time of such fluctuations.

The principal aim of this study is to elucidate whether these fluctuations have a random time-evolution or they rather evolve with a specific periodicity. A clarification of this issue would not only lead to a more detailed characterization of Minimally Conscious State but, more importantly, could be crucial for establishing a proper timing for performing behavioral assessments; such assessments would be thus performed during the identified maximum vigilance/awareness periods, ruling out those inconsistencies that render a correct disorders of consciousness diagnosis so challenging and possibly reducing the percentage of misdiagnosis (which is estimated at about $40 \%$ [7]).

At present, the gold standard tool for the diagnosis of disorders of consciousness in severely brain-injured patients is the Coma Recovery Scale-Revised, CRS-R, $[8,9]$. More specifically, criteria for the diagnosis of MCS minus or plus are based on evidence of non-reflex behavior (i.e., visual pursuit, localization to pain) or response to command respectively [3]. These awareness-related behaviors must be reproducible and last for an appropriate time-period to be considered significant for a MCS diagnosis. The clinical assessment of a patient's level of consciousness thus depends on inferences drawn from the observations of her/his behaviors, be they spontaneous or in response to specific requests. A major challenge in the behavioral assessment is that the patient's response may be affected not only by possible sensory deficits, aphasia or motor dysfunctions but also, as anticipated in the previous paragraph, by fluctuating levels of awareness/vigilance, which may result in an underestimation of the patient's cognitive capacity, leading to a misclassification of her/his clinical condition. The timing of the clinical assessment is therefore crucial for a correct assessment of the patient's residual cognitive abilities. At present, it is recognized that the absence of clinical signs of conscious behaviors per se cannot be taken as definitive proof of the absence of consciousness [10, 11]. Based on this evidence, the bedside behavioral evaluation has been integrated with complementary electrophysiology or neuroimaging-based approaches (i.e., positron emission tomography, functional MRI and EEG) with the aim of improving the diagnostic accuracy [12, 13]. Such ancillary assessments allow studying the brain responses at rest, during sensory stimuli and active tasks (such as mental imagery). But also here, fluctuations in vigilance can bias the observed results and lead to possible false negative findings [5]. A better understanding and monitoring of the temporal fluctuations of vigilance and awareness in patients with disorders of consciousness is hence highly needed.

Recent studies have shown that EEG spectral entropy is a reliable correlate of the level of consciousness both in non-clinical (sleep [14]), and clinical (anesthesia [15] and disorders of consciousness [16]) set-ups. Entropy quantifies the irregularity, complexity, or unpredictability of a signal; spectral entropy in particular gives an estimation of the uniformity of a signal's power spectral distribution: in general all conditions of low or absent consciousness are characterized, at the EEG level, by stereotyped signals yielding thus a low spectral entropy, whereas signals associated with full consciousness display a high degree of complexity (and a higher spectral entropy).

We employ long-duration (4-h) EEG recordings to capture both static but, more importantly, dynamic EEG features characterizing patients in MCS as compared to VS/UWS. We here investigate group-level and singlesubject differences in a cohort of 12 patients with disorders of consciousness (6 MCS and $6 \mathrm{VS} / \mathrm{UWS}$ ) assessing differences in (1) relative power in five frequency bands of interest (i.e., delta, theta, alpha, beta1 and beta2), (2) spectral entropy (i.e., mean and variability coefficients) and (3) spectral entropy fluctuations periodicity over time.

\section{Methods}

\section{Patients}

Twelve individuals diagnosed with disorders of consciousness were included in the study. Six patients had a diagnosis of MCS (three MCS plus) and six of VS/UWS based on CRS-R assessment [3,8]; the patients population had an age of $49 \pm 5$ (mean \pm standard error), three were females. Seven patients had traumatic and five non-traumatic etiologies. They were studied without sedative medication in a sub-acute or a chronic setting (i.e. more than 2 weeks after brain injury, time since insult was $69 \pm 17$ days, mean \pm standard error). EEG patterns of all subjects were devoid of continuous epileptiform activity, suppression or bust-suppression patterns. All patients were assessed in their usual clinical environment (i.e., nursing homes or rehabilitation hospitals). CRS-R assessments were performed by trained neuropsychologists on the day of the EEG recording as well as 2 days before and 2 days after. A further criterion for the inclusion of patients in the study was the stability over time of their clinical diagnosis (agreement between the three CRS-R assessments) as, given the small sample size, the reliability of the diagnosis was a crucial factor for ensuring the significance of the observed findings. The CRS-R has six subscales, including auditory, visual, motor and oromotor/verbal functions, communication, and level of arousal. Scoring is based on the presence or absence of specific behavioral responses to sensory stimuli. Clinical and demographical details of each patient are reported in Table 1 (the CRS-R scores reported 
Table 1 Clinical details of patients included in the study

\begin{tabular}{|c|c|c|c|c|c|}
\hline Patient ID & Age & Gender & Time since insult (days) & Etiology & $\begin{array}{l}\text { Coma recovery scale- } \\
\text { revised sub-scores }\end{array}$ \\
\hline $\mathrm{MCS}^{\mathrm{c}}$ & 48 & M & 43 & Non-traumatic (CVA) & $\mathrm{A} 3^{\mathrm{a}} \mathrm{V} 5^{\mathrm{a}} \mathrm{M} 5^{\mathrm{a}} \mathrm{OV} 2 \mathrm{C} 1^{\mathrm{a}} \mathrm{W} 2$ \\
\hline MCS2 & 36 & M & 163 & Traumatic & $\mathrm{A} 0 \mathrm{~V} 3^{\mathrm{a}} \mathrm{M} 0 \mathrm{OV} 1 \mathrm{C} 0 \mathrm{~W} 2$ \\
\hline $\mathrm{MCS}^{\mathrm{b}, \mathrm{c}}$ & 16 & $\mathrm{~F}$ & 25 & Traumatic & $\mathrm{A} 3^{\mathrm{a}} \mathrm{V} 4^{\mathrm{a}} \mathrm{M} 2 \mathrm{OV} 1 \mathrm{C} 0 \mathrm{~W} 2$ \\
\hline $\mathrm{MCS}^{\mathrm{b}, \mathrm{c}}$ & 53 & M & 62 & Traumatic & $\mathrm{A} 2 \mathrm{~V} 3^{\mathrm{a}} \mathrm{M} 2 \mathrm{OV} 2 \mathrm{C}^{\mathrm{a}} \mathrm{W} 2$ \\
\hline MCS5 & 62 & M & 111 & Non-traumatic (Anoxia) & $\mathrm{A} 0 \mathrm{~V} 3^{\mathrm{a}} \mathrm{M} 2 \mathrm{OV} 1 \mathrm{C} 0 \mathrm{~W} 2$ \\
\hline MCS6 & 31 & $\mathrm{~F}$ & 44 & Non-traumatic (CVA) & $\mathrm{A} 2 \mathrm{~V} 3^{\mathrm{a}} \mathrm{M} 2 \mathrm{OV} 2 \mathrm{C} 0 \mathrm{~W} 2$ \\
\hline UWS/VS1 & 62 & M & 35 & Non-traumatic (CVA) & A0 V1 M0 OV1 C0 W2 \\
\hline UWS/VS2 & 54 & $\mathrm{M}$ & 16 & Non-traumatic (anoxia) & A1 V0 M1 OV1 C0 W2 \\
\hline UWS/VS3 & 74 & $\mathrm{~F}$ & 15 & Traumatic & A0 V0 M1 OV1 C0 W1 \\
\hline UWS/VS4 ${ }^{\mathrm{b}}$ & 32 & M & 186 & Traumatic & A0 V1 M1 OV1 C0 W2 \\
\hline UWS/VS5 & 61 & M & 119 & Non-traumatic (anoxia) & A1 V1 M2 OV1 C0 W2 \\
\hline UWS/VS6 & 61 & $\mathrm{M}$ & 15 & Non-traumatic (encephalitis) & A0 V1 M2 OV1 C0 W2 \\
\hline
\end{tabular}

$A$ auditory, $V$ visual, $M$ motor, $O V$ oromotor/verbal functions, $C$ communication, $W$ level of wakefulness [8]

a Scores fulfilling MCS criteria

b Patients not included in the study of Cologan et al. [17]

c MCS plus diagnosis

in the table refer to the assessment performed on the day of the EEG recording). The study was approved by the Ethics Committee of the Faculty of Medicine of the University of Liège, and written informed consent was obtained from the patients' legal representatives. EEG sleep recordings from 9 patients (4 MCS and 5 VS/UWS, see Table 1) have been published elsewhere [17].

\section{EEG recordings}

The polygraphic recordings were performed using a V-Amp16 amplifier (Brain Products, Germany). Signals from twelve electroencephalographic (EEG) electrodes placed according to the 10-20 system (F3, Fz, F4, C3, Cz, $\mathrm{C} 4, \mathrm{~T} 3, \mathrm{P} 3, \mathrm{Pz}, \mathrm{P} 4, \mathrm{Oz}$ ) were acquired, along with chin electromyography (EMG), and electro-oculography signals (EOG in crossed montage). Eleven recordings lasted for $24 \mathrm{~h}$ starting at 5:30 pm (see Cologan et al., 2013, [17]) and one lasted for $16 \mathrm{~h}$ (starting at 5:30 p.m.). All signals were acquired referenced to the nasion with a sampling rate of $500 \mathrm{~Hz}$ and impedances were kept below $5 \mathrm{k} \Omega$ at the start of the recordings. We retained and analyzed only the first $4 \mathrm{~h}$ of recordings (5:30-9:30 p.m.) both to have a good and stable signal quality (as the impedance of channels gradually increased over time), which is a mandatory condition for a reliable evaluation of spectral entropy (note that a random noise time series would yield by definition the highest spectral entropy), and because patients in the first $4 \mathrm{~h}$ were prevalently in an eyes-open condition, as verified by visual inspection from the clinicians (rooms lights were switched-off at about 10:30 pm). The prevalence of the eyes-open condition (i.e. eye blinks) and the absence of relevant markers of NREM sleep, both related to the EEG (i.e., spindles, K-complexes and Sleep Slow Oscillations), to the EOG (disappearance/and or reduction of eye movements) and to the EMG (disappearance or consistent lowering of muscular activity) were confirmed by signals' offline visual inspection. Two subjects yielding sufficiently noise-free traces for all the recording time (at least when considering $\mathrm{Fz}$ derivation), were selected for a proof-of-concept $24 \mathrm{~h}$ analysis (patients MCS3 and VS/UWS5).

\section{Signal pre-processing}

All the analyses were performed in Matlab (Mathworks, Natick, MA, USA). EEG, EMG and EOG signals were band-pass filtered between 1 and $45 \mathrm{~Hz}$. The filters were applied on signals both in the forward and reverse directions to avoid time biases, and each recording was segmented into $4 \mathrm{~s}$ consecutive epochs. Epochs where the voltage range (peak-to-peak amplitude of the signal) of either EEG or EOG channels exceeded $100 \mu \mathrm{V}$ were excluded from the analysis. EEG traces were then visually scanned, and epochs contaminated by residual artifacts were discarded.

It is worth underlining that all the EEG recordings, regardless of the CRS-R classification of the specific patient, were highly contaminated by muscular artifacts. Even if noisy epochs were removed by visual inspection, an influence of electromyographic activity on the EEG features could still not be entirely ruled out. In order to 
ensure the robustness of the presented findings, all the analyses were conducted only for midline derivations (Fz, $\mathrm{Cz}$ and $\mathrm{Pz}$ ), which among the EEG channels were the less contaminated by EMG artifacts (as verified by a visual inspection of the EEG traces pertaining to each patient). $\mathrm{Oz}$ was excluded from the analyses as its signal quality was already poor after $1 \mathrm{~h}$ of recording for most of the patients: the recordings were performed at bedside and the continuous friction of the scalp on the pillow caused by head movements resulted either in a fast degradation of the scalp-electrode contact (with progressively higher contact impedance) or in a fluctuating contact which caused highvoltage sweeps on the EEG trace. To further ensure that differences between the two groups of patients were due to their brain electrical activity and not to difference in muscle activity or movement, the analyses performed on the EEG were replicated also for the chin EMG signal. The rationale of this approach stands on the following: if the same putative difference between MCS and VS/UWS patients found for a feature extracted from the EEG signal was also found on the same feature extracted from the EMG, this would cast doubt on the cortical origin of the EEG finding. If on the other hand, no difference was found for the EMG signal, this supports the cortical origin of the observed result. As a last precaution, to avoid as much as possible any contamination of EMG activity on EEG data, both beta2 band activity and spectral entropy were estimated by choosing an upper frequency limit of $25 \mathrm{~Hz}$ instead of the classical $30 \mathrm{~Hz}$.

\section{Between-group statistical analysis}

Differences in gender and etiology (traumatic versus nontraumatic) were assessed using Fisher-exact test. All other between-group statistical analyses (including differences in age and time since insult) were conducted calculating their $t$-values from unpaired $t$-tests; for each between-group comparison, the $p$-value was estimated applying a randomization test on the $t$-value [18] as, given the small sample size, no reliable assumption could be made on the distributions' shapes. Five hundred random relabeling of the patients were made, assigning within each randomization one or more patients of the former group to the latter one and vice versa, under the null-hypothesis of no significant difference between the two groups. For each randomization, the $t$-value related to the unpaired $t$-test was estimated. At the end of the procedure, a distribution of $t$ values under the null-hypothesis of no between-groups significant differences was obtained. The test significance was then estimated as the ratio between the number of randomly generated $t$-values exceeding the real one (all $t$ values were taken in absolute value for two-tails significance assessment) and the total number of randomizations.
Descriptive statistics of each feature (unless otherwise stated) were expressed by the distribution mean and its $95 \%$ confidence interval (the confidence interval was estimated on the basis of 500 bootstraps of the original dataset). This procedure was applied for all the statistical tests described in the manuscript (with the exception of Fisher-exact tests).

\section{Signal analysis}

As a first step, we verified that both the number of retained epochs and the mean time distance between consecutive epochs (the distance was computed between the beginnings of the retained contiguous epochs) were consistent when comparing the MCS to the VS/UWS group.

For each patient, channel (EEG or EMG) and epoch, both the total and relative powers in five bands of interest were extracted: delta $(1-3.75 \mathrm{~Hz})$, theta $(4-7.75 \mathrm{~Hz})$, alpha $(8-11.75 \mathrm{~Hz})$, beta1 $(12-17.75 \mathrm{~Hz})$ and beta2 $(18-24.75 \mathrm{~Hz})$. For each channel and epoch, the Power Spectral Density was estimated by applying a Hammingwindowed Fast Fourier Transform, and the relative power in each band was obtained as the ratio between the total power in the band and that in the $1-25 \mathrm{~Hz}$ range.

The spectral entropy was thus defined as

se $=-\frac{\sum_{k=1}^{K}\left(P_{f_{k}} \log _{2} P_{f_{k}}\right)}{\log _{2} K}, 1 \mathrm{~Hz} \leq f_{k} \leq 25 \mathrm{~Hz}$,

$P_{f_{k}}$ denoting the normalized power spectral density at the $f_{k}$ frequency.

The retained $4 \mathrm{~s}$ epochs were then grouped into $5 \mathrm{~min}$ consecutive intervals, and both for the total and relative band powers and for spectral entropy mean, standard deviation and coefficient of variation $\left(C_{\mathrm{v}}=\sigma / \mu\right)$, the values related to each interval were estimated by averaging the epoch values pertaining to each interval, thus obtaining 48 time samples for each parameter.

Features of interest were then grouped in four datasets (Fz, $\mathrm{Cz}$ and $\mathrm{Pz}$ and EMG). Differences between MCS and VS/UWS groups were assessed for each feature by calculating its $t$-value (unpaired $t$-test); the $t$-value significance was evaluated using the randomization approach explained in the "Between-group statistical analysis" section.

If needed (i.e. if at least one of the tests related to the dataset was significant), the significance of tests pertaining to the dataset were corrected applying the False Discovery Rate, FDR, [19], with the aim of controlling type I error rate.

For each patient the time series of (1) spectral entropy (Fz, $\mathrm{Cz}$ and $\mathrm{Pz}$ ); (2) log-transformed delta, theta, alpha, beta1 and beta2 total power (Fz channel); (3) log-transformed EOG and EMG raw power $(1-40 \mathrm{~Hz})$ were collected. It is worth underlining that for the latter series (and 
only referred to time series analyses), only $4 \mathrm{~s}$ epochs affected by movement artifacts were discarded, retaining thus muscular and eye activities. In line with spectral entropy, EEG, EOG and EMG power time series were obtained by grouping the retained $4 \mathrm{~s}$ epochs into $5 \mathrm{~min}$ consecutive intervals.

Each time series was submitted to a wavelet analysis with a Morlet basis to highlight the contribution of oscillations at different scales/frequencies (i.e., with different periodicities) to the series' time-course (the sampling frequency of the time-series being $f_{\mathrm{s}}=\frac{1}{5 \times 60}=0.033 \mathrm{~Hz}$ ).

The number of wavelets cycles of the Morlet function $\left(\omega_{0}\right)$ was set to 4 and kept constant at all scales. The wavelets transform of a time-series $x_{\mathrm{n}}$ at a scale $s$ and timepoint $n$ is estimated as the convolution of $x_{\mathrm{n}}$ with a scaled and translated version of the mother wavelet:

$W_{\mathrm{n}}(s)=\sum_{\mu=0}^{N-1} x_{\mu} \psi^{*}\left[\frac{(\mu-n) \delta t}{s}\right]$

$N=48$ is the number of time samples of the time series, $\delta t=\frac{1}{f_{s}}$ the time lapse between consecutive samples and * indicates the complex conjugate operator. An approximation of the continuous wavelet transform can be obtained performing the convolution of Eq. (1) $N$ times for each scale. Taking advantage of the Discrete Fourier Transform, the $N$ convolutions can be conducted simultaneously and on the basis of the convolution theorem, the wavelet transform can then be rewritten as:

$W_{\mathrm{n}}(s)=\sum_{k=0}^{N-1} X_{k} \Psi^{*}\left(s \omega_{k}\right) e^{i \omega_{k} n \delta t}$

upper-case letters denoting the Discrete Fourier Transforms of the functions and $\omega_{k}$ being the angular frequency. As the Morlet basis is non-orthogonal, an arbitrary set of scales can be used. Scales were then expressed as fractional powers of two, in agreement with Torrence and Compo, [20]: $s_{j}=s_{0} 2^{j \delta j}$, where $j$ was chosen in order to cover the scales (and corresponding frequencies) of interests; $s_{0}$ was set equal to $2 \delta t$ to satisfy the condition on the equivalent Fourier period (which must be $\geq 2 \delta t$ ) as the following equation holds for $\omega_{0}=4$ :

$T \sim 1.52 \mathrm{~s}$

As empirically demonstrated by Torrence and Compo, [20], the maximum value of $\delta j$ giving an adequate sampling in scale is of about 0.5 ; in the present study a finer scale resolution was chosen setting $\delta j=0.1$. Finally the amplitude spectrum at a given frequency $f_{i}$ can be expressed as:

$A\left(f_{i}\right)=\left|W_{\mathrm{n}}\left(\frac{1}{1.52 s_{i}}\right)\right|$ or as a function of the corresponding period:

$A\left(T_{i}\right)=\left|W_{\mathrm{n}}\left(1.52 s_{i}\right)\right|$.

For easiness of interpretation, instead of referring to the amplitude spectrum as a function of the oscillation frequency (4), we will here refer to the amplitude spectrum as a function of the oscillation period (5).

For each patient and feature, the mean amplitude spectrum distribution as a function of the period, was estimated by averaging the amplitude spectrum along the $240 \mathrm{~min}$ (48 samples) time window.

The spectral entropy time-series analysis for the two selected $24 \mathrm{~h}$ recordings were performed with the same approach described above (in this case 288 samples with a time-step of 5 min were collected), starting from the visual scoring performed on EEG, EOG and EMG traces which aimed at the identification of NREM sleep epochs.

For each patient, the period corresponding to the oscillation showing the maximum contribution to the spectral distribution of $\mathrm{Fz}$ spectral entropy was extracted. The corresponding oscillation in the time domain was then computed by applying the inverse wavelet transform on the wavelet coefficient related to the periodicity of interest. It is worth underlining that each time-series was zero-padded in order to resolve oscillations with periodicities up to $120 \mathrm{~min}$.

For each feature and diagnostic group (MCS or VS/ UWS), the mean spectrum was calculated by averaging the group patient's spectra. For each feature and group, a repeated measures ANOVA on the mean amplitude spectrum with periodicity as a within-subject factor (26 levels, see Supplementary Material for the considered periodicities) was conducted to verify whether oscillations with different periodicities showed or not significantly different amplitudes. The $F$-value significance was assessed on the basis of 500 randomizations. Whenever a significant periodicity effect was found, the significance of the periodicity distribution's peak was evaluated as follows: under the null-hypothesis of no significant periodicity-effect, for each patient, the spectral amplitude corresponding to one periodicity could be randomly assigned to another periodicity. Under this hypothesis, 500 surrogate 6-elements series (corresponding to the 6 patients included in the group) were created assigning for each patient a randomly chosen value among those pertaining to his/her amplitude spectrum, to the peak periodicity and for each series the mean value was calculated. The periodicity peak significance was assessed by computing the ratio of mean values derived from the surrogate series exceeding the real peak value, and the total number of surrogates (500).

The peak periodicities of both Fz power in the five bands of interest and of EMG and EOG power were then compared to those found for $\mathrm{Fz}$ spectral entropy. For each 
patient, the time-courses of log-transformed EOG, EMG and $\mathrm{Fz}$ bands powers were then correlated (Pearson's correlation) to the spectral entropy time-course, extracting their respective $r$-values. Prior to correlation, each timecourse was tested for normality (Shapiro-Wilk test [21]) and as about $70 \%$ of the distributions did not stand the test, $r$-values' significance was assessed performing randomization tests on the $r$-statistics (500 permutations). The estimated $p$-values were corrected applying the FDR approach [19].

As a final step, CRS-R total scores were correlated (Pearson's correlation) to spectral entropy features (mean, standard deviation, coefficient of variation and periodicity), considering both the whole cohort of patients regardless of their CRS-R diagnosis, and the single groups (MCS and VS/UWS). $r$-values' significances were again assessed by using randomization tests on the $r$-statistics (500 permutations) and when appropriate, FDR correction was applied.

\section{Results}

MCS did not differ from VS/UWS group either by age (41, $28-55$ versus $57,41-67$ years, $p<0.13)$, time since insult (75, 43-131 versus 64, 22-136 days, $p<0.76$ ), gender or etiology ( $p<1$ for both) as apparent from Table 2. No difference was observed between MCS and VS/UWS patients either for the total number of retained EEG epochs $(p<0.52)$ or for the time distance between contiguous epochs $(p<0.27$, see Table 2$)$.

\section{Band relative power}

Relative power spectra of the Fz signal were computed for each patient (Fig. 1 and Table A of Supplementary
Material). Delta power was higher for the VS/UWS when compared to the MCS group $(p<0.005)$ whereas theta, alpha and beta1 bands showed higher power in the MCS group as compared to the VS/UWS group $(p<0.05$, $p<0.005$ and $p<0.05$ respectively, see Fig. 1 and Table A of Supplementary Material). Analogous results were obtained when considering relative power spectra of $\mathrm{Cz}$ and $\mathrm{Pz}$ signals (Tables $\mathrm{B}, \mathrm{C}$ of Supplementary Material). For the latter channel, contrary to the formers, beta 1 band showed only a tendency towards significance $(p<0.1)$. Two main reasons lead us to the choice of relative powers instead of total powers when dealing with between-groups comparison:

- Rendering the analyses results consistent and comparable to those of previously published studies $[18,19]$.

- When dealing with sparse electrode arrays (three electrodes in our case), the positioning of the electrodes is a crucial issue as it should be highly consistent between subjects to obtain non-biased comparisons of non-normalized powers (this becomes a minor issue when using high-density electrode arrays).

Relative powers in delta, theta, alpha, beta1, beta 2 bands were estimated also for the EMG channel. No betweengroups difference was found for any of the considered bands and consequently no FDR correction was applied, as the minimum $p$-value found was $p=0.428$ related to theta band comparison (see Table D of Supplementary Material for all other results), supporting the assumption that EEG findings cannot be explained by between-groups differences in movement or muscle activity (note that the estimation of relative power within delta and theta band was carried out also for the EMG signal only for the sake of completeness, as surface electromyographic activity has

gender and etiology (number of patients per category). In the last column, the results of the statistical analyses are reported
Table 2 Descriptive statistics of each group are reported for age, time since insult, number of retained epochs and time-distance between consecutive epochs (mean and $95 \%$ confidence interval) and

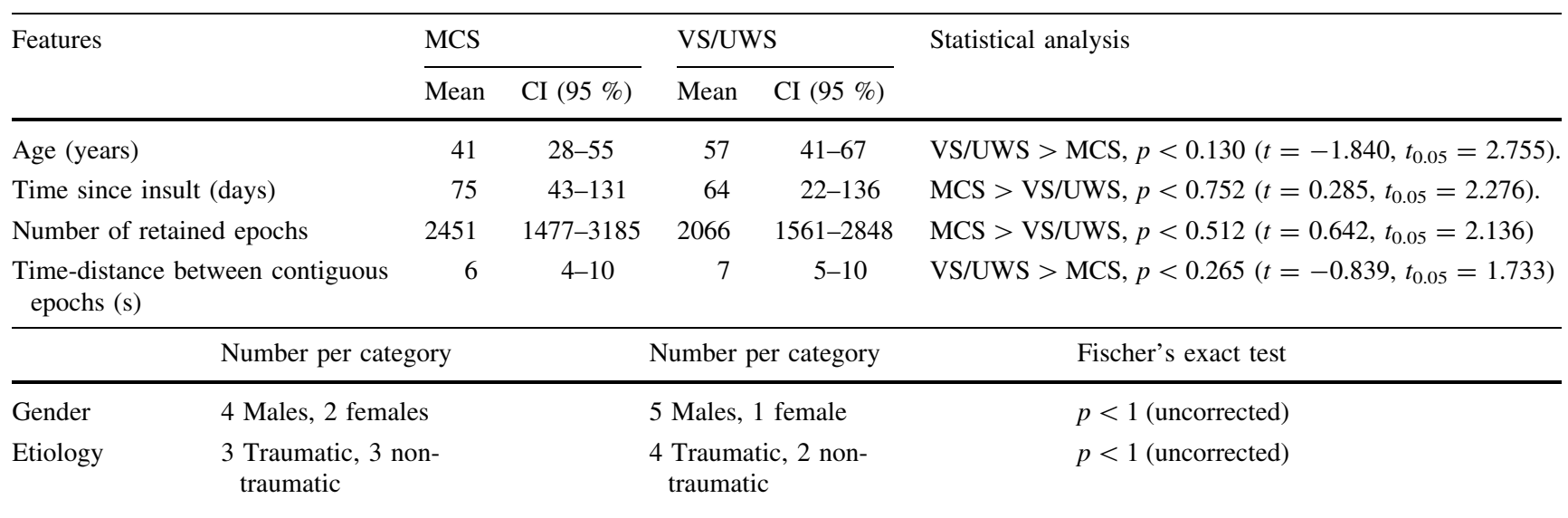


Fig. 1 Descriptive statistics of relative powers (mean and $95 \%$ confidence interval) are depicted for each band of interest and each group. The estimates refer to the $\mathrm{Fz}$ derivation. Red arcs indicate significance at $p<0.005$, whereas black arcs significance at $p<0.05$ (all the presented $p$ values are FDR-corrected)
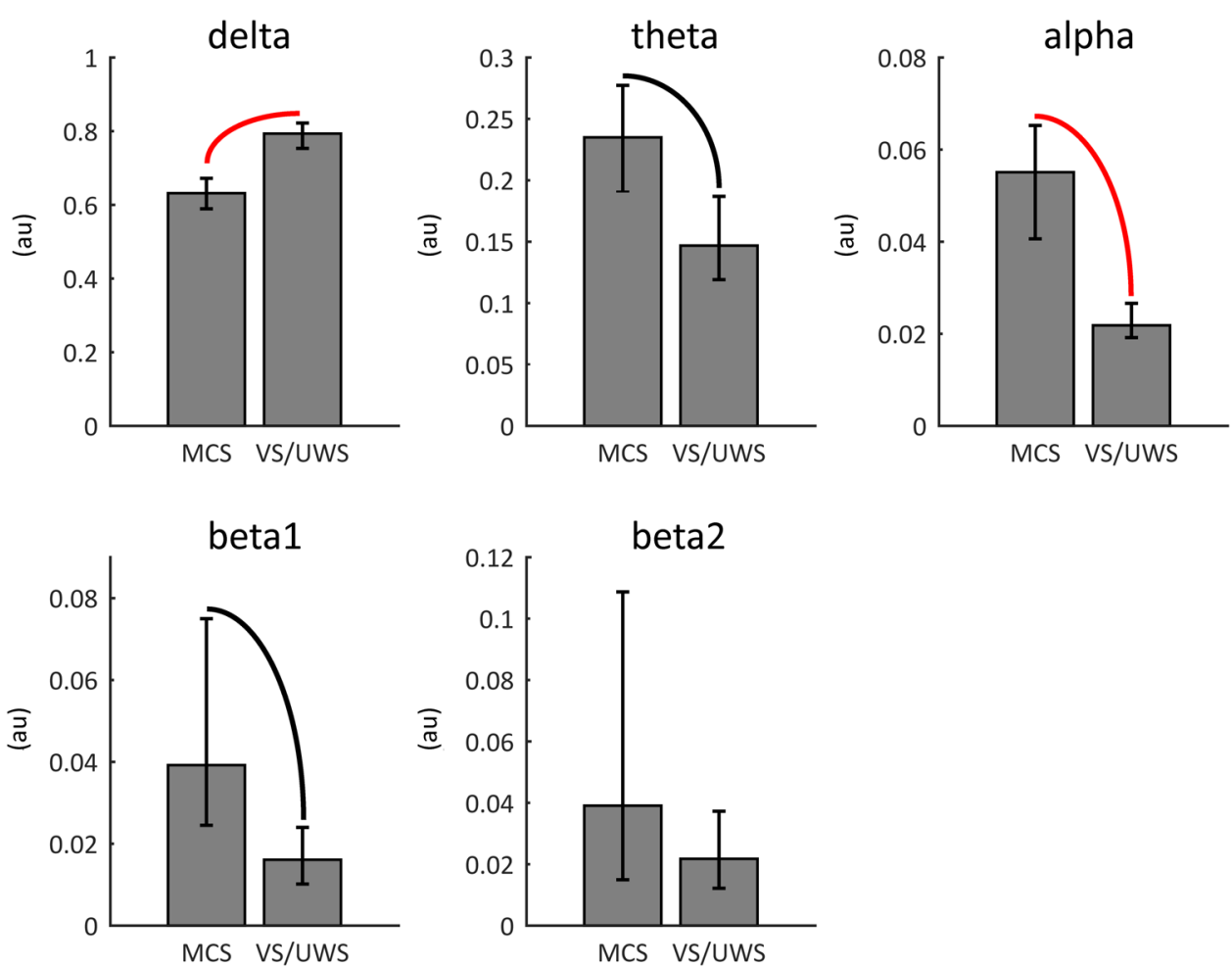

negligible contributions at frequency lower than $10 \mathrm{~Hz}$, [17]).

\section{Spectral entropy}

The spectral entropy time-course was estimated for $\mathrm{Fz}, \mathrm{Cz}$ and $\mathrm{Pz}$ derivations and for the EMG channel with a time step of $5 \mathrm{~min}$. With regard to $\mathrm{Fz}$, MCS mean spectral entropy was higher than that of the VS/UWS group (0.680 versus $0.592, p<0.005$, see Fig. 2 and Table A, Supplementary Material). MCS patients had a higher standard deviation and coefficient of variation when compared to the VS/UWS group (0.016 and 0.024 for MCS, 0.004 and 0.007 for VS/UWS, $p<0.005$ for both tests). Analyses on $\mathrm{Cz}$ and $\mathrm{Pz}$ channels confirmed the results obtained for $\mathrm{Fz}$ (Tables B, C of Supplementary Material). When considering EMG-derived spectral entropy parameters, no difference was found between the two groups (the minimum $p$-value found was $p=0.278$ ); this result holds both for the spectral entropy estimated between 1 and $25 \mathrm{~Hz}$ and for the one estimated in the $10-25 \mathrm{~Hz}$ range, see Table $\mathrm{D}$ of Supplementary Material).

\section{Wavelet decomposition of spectral entropy time-courses}

For each patient, the time-courses of spectral entropies were submitted to a wavelet analysis in order to identify, if present, dominant oscillatory components (at different frequencies/periods) contributing to their time-evolution. Fig. 3 and 4a highlight how each MCS patient was characterized by a dominant oscillatory component, MCS periodicities ranging from 57 to $80 \mathrm{~min}$. Results of the wavelet decomposition were synthesized, for easiness of interpretation, by computing the mean value across time for each periodicity (Fig. 4a; Fig. A, B, Supplementary Material).

For each patient, the oscillation corresponding to the peak of the distribution was identified and its timecourse was estimated by applying the inverse wavelet transform to its wavelet coefficient (Fig. 5; Fig. C of Supplementary Material). All MCS patients had an apparent main oscillation (i.e., the one contributing the most to the spectral entropy time-course), which lasted for the entire recording $(4 \mathrm{~h})$ with a period included in the 53-80 min range (see Fig. 4, 5; Fig. A-C, Supplementary Material).

On the other side, the VS/UWS group showed a higher intra-group variability with small oscillations (ranging in periodicity from 25 to $106 \mathrm{~min}$ ). One VS/UWS patient (VS/UWS1) did not show any peak in the 20-120 min range. The same analyses were performed also for $\mathrm{Cz}$ and $\mathrm{Pz}$ and yielded results in line with those found for $\mathrm{Fz}$ (see Fig. A, B, Supplementary Material). MCS patients showed main oscillations included in the 53-80 min range, whereas the VS/UWS were still characterized by a high intra-group variability with small oscillations (range 35-120 min both for $\mathrm{Cz}$ and $\mathrm{Pz}$ ). Of note, only one subject within the VS/ 
Fig. 2 Descriptive statistics of spectral entropy measures for $\mathrm{Fz}$ channel are presented. In the left plot the means, in the central plot the standard deviations and in the right one the coefficients of variation of respectively MCS and UWS groups are depicted. Red arcs denote statistically significant differences $(p<0.005$ after FDR-correction). Black vertical lines denote the $95 \%$ confidence interval on the mean
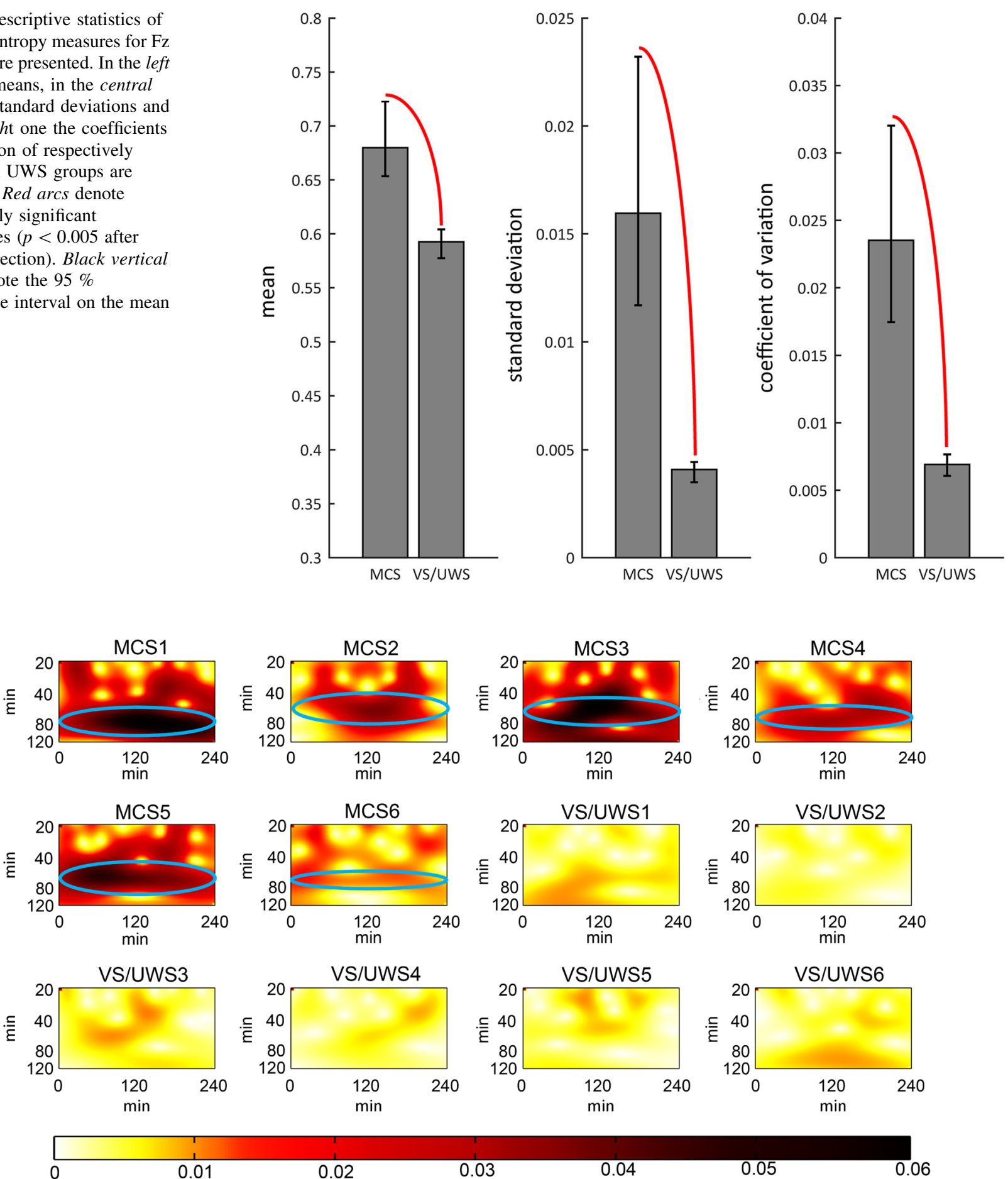

0.02

\section{sa}

Fig. 3 The time-course of the $\mathrm{Fz}$ spectral entropy amplitude spectrum (sa) is depicted for each subject, the $x$-axis identifying the time-course (i.e., $240 \mathrm{~min}$ of EEG recording) and the $y$-axis the periodicities of the oscillations composing the spectrum time-course.

UWS group maintained comparable periodicities for the three electrodes (33-37 min, VS/UWS3). On the other side, subjects VS/UWS5 and 6 showed a periodicity in line
Colors from white to dark-red identify progressively higher contributions to the time-series of spectral amplitude variations. Blue ellipses denote relevant long-lasting oscillatory components observed in MCS but not in VS/UWS

with that of compatible with those found in the MCS group (around 55 min for the former both for $\mathrm{Cz}$ and $\mathrm{Pz}$ derivations, and $70 \mathrm{~min}$ for the latter, $\mathrm{Cz}$ electrode). 

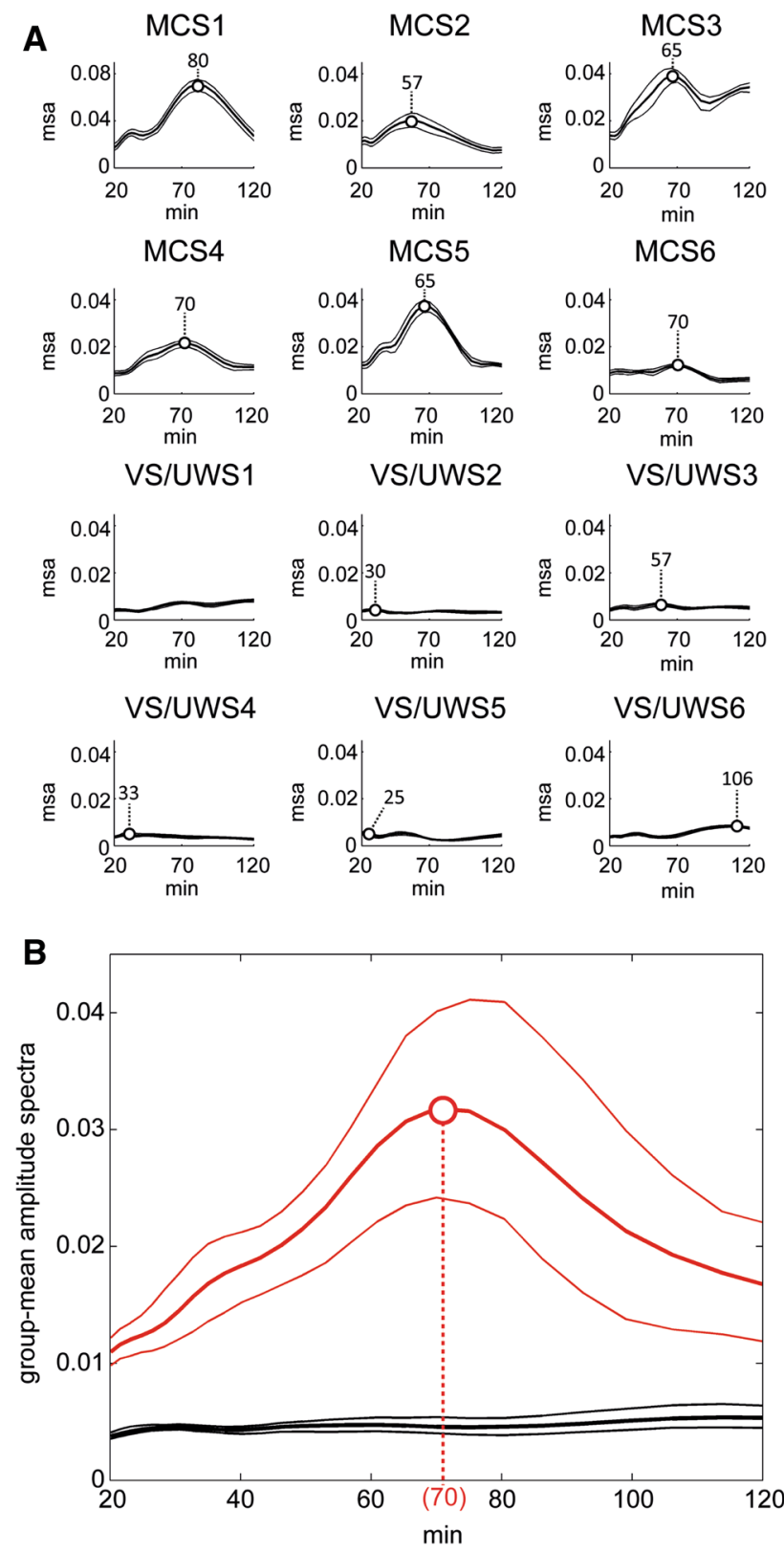

Fig. 4 Panel a For each subject, the mean contribution of oscillations with periodicities ranging from 20 to $120 \mathrm{~min}$ to the $\mathrm{Fz}$ spectral entropy time-variations are depicted (MSA). Note the presence of a well-defined peak in the distribution for all subjects within the MCS group (the peak is less prominent for MCS6). In each sub-plot, the mean contribution is enclosed within the limits of the $95 \%$ confidence interval time-courses. Note that for visualization purposes MCS1 has a different scale with respect to the other subjects. Panel b The group-level mean amplitude spectra (related to $\mathrm{Fz}$ spectral entropy) of both MCS (red lines) and VS/UWS patients (black lines) are depicted. The course of each spectrum as a function of the oscillation period is described by three lines: two thin lines representing the $95 \%$ confidence interval on the mean and the thick line between them representing the mean series. Note that for MCS group $(n=6)$ a significant peak occurs at $70 \mathrm{~min}$ (red dot) while no significant peak could be identified for the VS/UWS group $(n=6)$
Figure 6 (first row), shows the group-averaged amplitude spectra: the MCS group is characterized by a welldefined peak corresponding to the oscillatory component at $70 \mathrm{~min}$ holding for all three derivations, whereas no meaningful peak could be identified in the VS/UWS group.

Repeated measures ANOVA with periodicity as a within-subject factor (26 levels, corresponding to the number of periodicities/frequencies bins estimated in the wavelet analysis, see Supplementary Material) identified a periodicity-effect in the MCS group coherently for all three EEG derivations $(p<0.005)$ but not in the VS/UWS group $(p=0.971, p=0.072$ and $p=0.924)$. A significant peak with a 70 min periodicity was identified in the MCS group $(p<0.005)$ whereas no significant peak could be found for VS/UWS group (see Fig. 6; Table E of Supplementary Material).

\section{Identification of potential factors driving spectral entropy fluctuations}

The observed spectral entropy fluctuations could be affected by consistent vigilance shifts (i.e. by the occurrence of periods of drowsiness or sleep), thereby strongly biasing the observed results. The possible influence of vigilance shifts on spectral entropy fluctuations was evaluated exploiting two complementary approaches:

1. Evaluation of the periodicities of $\mathrm{Fz}$ bands power and EOG-EMG power time-courses.

2. Within-subject correlations between spectral entropy time-course and power time-courses related to EEG bands of interest and to EOG-EMG power.

In this framework, total powers and not relative powers were used as the analyses were performed at a withinsubject level with the advantage that the correlation between spectral entropy and total power within a specific band (contrary to its relative power) would not be affected by the power content of other bands.

As apparent from Fig. 6 and Table E of Supplementary Material, the only feature sharing the same periodicity of spectral entropy, at least for the MCS group, was beta2 (when interpreting the figure, keep in mind that red circles indicate significant peaks or maxima, whereas black circles non-significant ones). A main periodicity of $60 \mathrm{~min}$ was found for theta (MCS group) although it was proven nonsignificant ( $p<0.07$, uncorrected) when compared to other periodicities (Table E, Supplementary Material). In agreement with these findings, we observed that, at the single subject level, features sharing the same periodicity of the spectral entropy were heterogeneous and no coherent pattern could be found among patients (Table F, Supplementary Material). 
Fig. 5 The Fz spectral entropy time course is depicted for each subject (black traces, SE stands for spectral entropy). For easiness of interpretation and only for the MCS subjects, the time-course of the main oscillation identified in the wavelet analysis is superimposed to the spectral entropy time-course (red traces). In order to be represented on the same scale of the spectral entropy, the timecourse of each oscillation derived from the inverse wavelet transform was normalized to its range and multiplied to the range of the detrended original signal. As a last step, the mean value of the original signal was added to the oscillation time-course
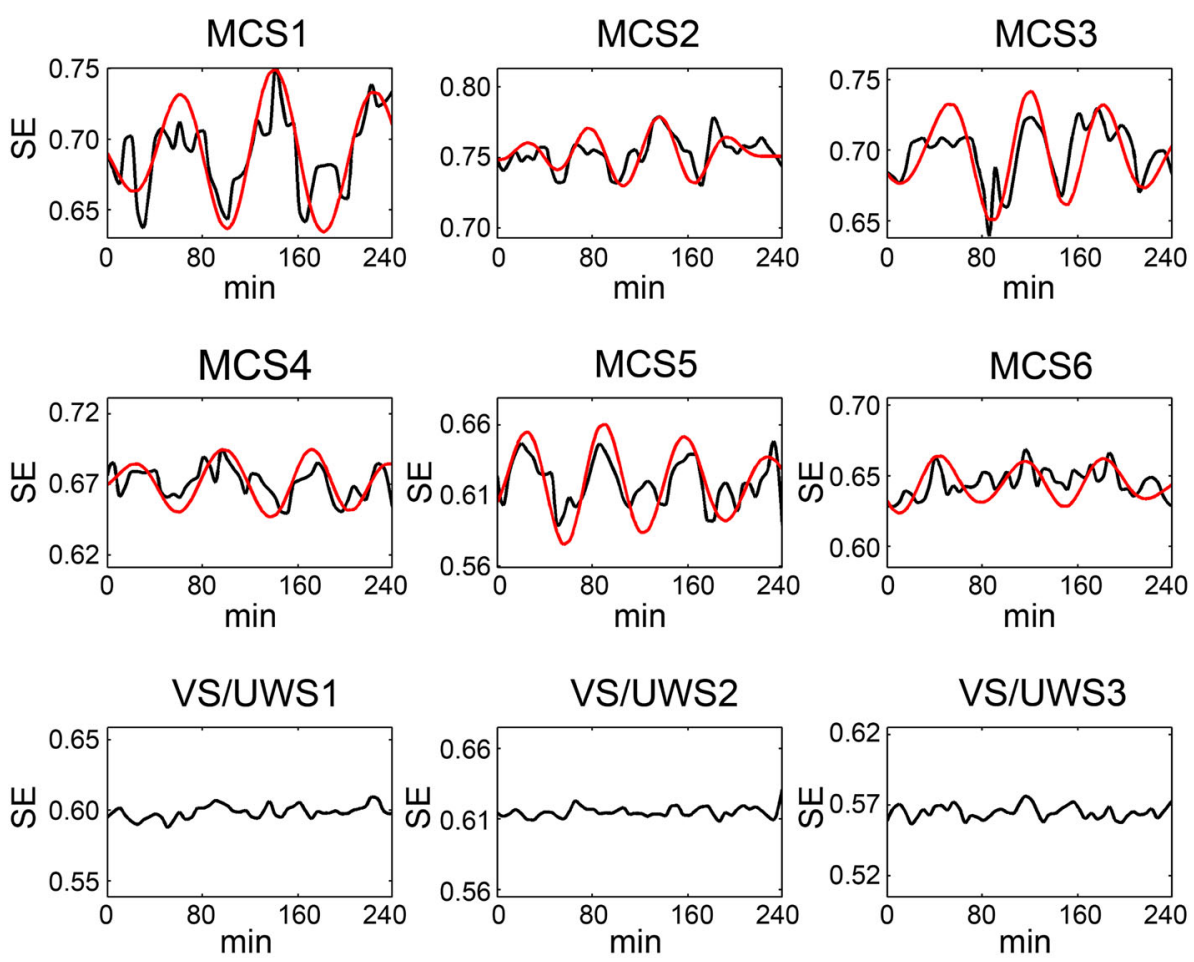

VS/UWS4
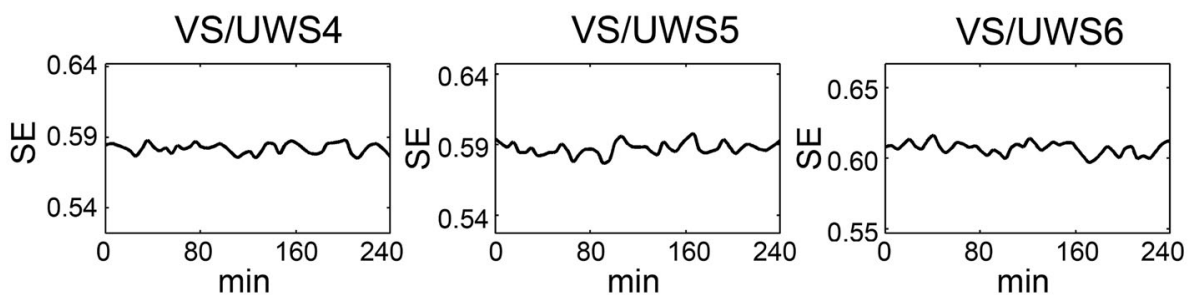

No significant correlation could be found between spectral entropy and either EOG or EMG power (with the exception of VS/UWS1 which showed a significant anticorrelation with EOG power). When dealing with EEG bands powers a heterogeneous set of significant correlations and anti-correlations were found (Fig. D of Supplementary Material) and also in this case no specific pattern of correlation could be found, even when considering the MCS group only as:

- Significant anti-correlations with delta power were found for MCS3, MCS4, MCS5 and VS/UWS5 patients ( $p<0.01$ for all of them).

- Significant correlations with theta power were found for MCS1, MCS5 and VS/UWS3 ( $p<0.05$ for the former and $p<0.01$ for the latter). We also observed significant anti-correlations when considering MCS3, MCS4, MCS6 and VS/UWS5.

- Correlations with alpha power were significant $(p<0.01)$ for MCS1 and VS/UWS3.
- Both beta1 and beta2 yielded significant correlations coherently for MCS1, MCS6 and VS/UWS6.

\section{Correlations between spectral entropy features and CRS-R total scores}

Significant correlations between spectral entropy variability indices (i.e. standard deviation and coefficient of variation) and CRS-R total scores (Fig. E of Supplementary Material) were found when considering the whole cohort of patients regardless of their diagnosis ( $p<0.006$ for both) and a tendency towards significance was apparent for the mean spectral entropy $(p<0.06)$. The same correlation analyses where conducted also at the single group level: when dealing with VS/UWS patients, no significant correlation was found, whereas a significant positive correlation was found between CRS-R total scores and spectral entropy periodicities $(p<0.02)$. Results regarding correlations analyses can be found in Table I of Supplementary Material. 

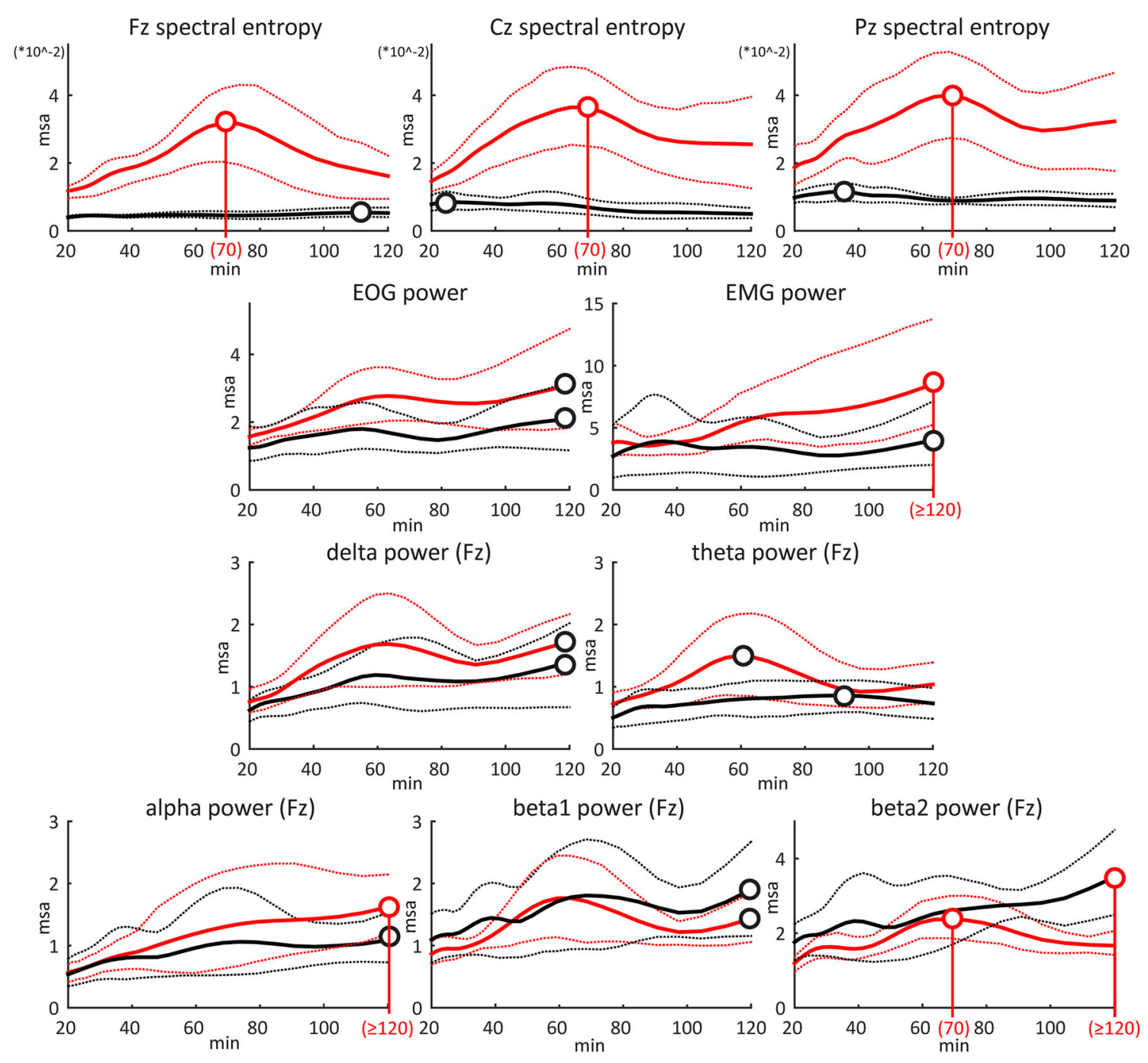

Fig. 6 The group-level mean amplitude spectra of both MCS (red lines) and VS/UWS patients (black lines) are depicted. The course of each spectrum as a function of the oscillation period is described by three lines: two thin lines representing the $95 \%$ confidence interval on the mean and the thick line between them representing the mean

\section{Comparison between sub-acute and chronic VS/UWS patients}

The VS/UWS group included three sub-acute patients (time since insult 15-16 days), thereby introducing a possible confounding factor in the group analysis. The coherence between the two VS/UWS sub-groups was assessed confronting the single subject's descriptive statistics of relative powers, spectral entropy mean, standard deviation and coefficient of variation and spectral entropy periodicities.

When considering Fz relative powers, we found comparable delta values between the two sub-groups; one of the chronic patients (189 days since insult), had a substantially higher delta power when compared to all other series. Significant peaks and maxima are denoted by red circles whereas non-significant ones by black circles. First row refers to $\mathrm{Fz}$, $\mathrm{Cz}$ and $\mathrm{Pz}$ spectral entropies, second row to EOG and EMG powers, third and fourth rows to power within the five bands of interest estimated on the Fz signal

patients, which resulted in really low power values when considering the other four bands (it is worth underlining that when considering $\mathrm{Cz}$ and $\mathrm{Pz}$ relative powers this potential outlier disappears, see Table F of Supplementary Material). The inspection of relative power in the other bands did not highlight any systematic difference between the two sub-groups (see Fig. F and Table G of Supplementary Material).

Regarding Fz spectral entropy features (Fig. G of Supplementary Material), maximum and minimum mean values where found in the sub-acute group (VS/UWS2 and VS/UWS3 patients). The sub-acute sub-group had spectral entropy mean values in the $0.57-0.61$ range, whereas the chronic sub-group in the 0.59-0.60 range. When considering $\mathrm{Cz}$ and $\mathrm{Pz}$ derivation ranges for the sub-acute were 
0.54-0.61 and 0.53-0.57, whereas those related to chronic patients $0.58-0.60$ and $0.56-0.61$ (Table $\mathrm{H}$ of Supplementary Material). All VS/UWS patients independently form the time since insult had spectral entropy mean values well-below those of the MCS group. Also when considering either standard deviations or coefficients of variation no difference was apparent between the two sub-groups (the results holds for all the three derivations). As a last point, we examined if any difference in spectral entropy periodicity could be found in the two sub-groups. As apparent from Fig. H, Supplementary Material, the two sub-groups are characterized by the same inconsistencies in periodicity and similar between-subject variability.

\section{Spectral entropy time course and periodicity in $24 \mathrm{~h}$ recordings}

As a proof-of-concept of the feasibility of spectral entropy fluctuation analysis on $24 \mathrm{~h}$ recordings, we herein report the results pertaining to two exemplary subjects (MCS3 and VS/UWS5). MCS3 showed, when combining the visual scoring (based on EEG, EOG and EMG traces) with the examination of delta power and spectral entropy timecourses (Fig. I, Supplementary Material), a cycled sleep period starting at about 22:30 and ending at around 6:00. During this period delta activity reached its maxima and showed a tight anti-correlation with spectral entropy and at least two sleep cycles could be detected. From 6:00 to about 9:30 the patient showed a fragmented EEG with short-lasting periods resembling NREM sleep alternated by arousals and/or patterns resembling REM sleep. When considering the whole $24 \mathrm{~h}$ period, a main oscillation lasting about $7 \mathrm{~h}$ could be detected (Fig. I, Supplementary Material, third and fourth rows). The 65 min periodicity found in the first $4 \mathrm{~h}$ of recordings, was found again after the sleep period (i.e. when analyzing the time-course from 9:30 to 17:30). Regarding the VS/UWS subject, while his/ her spectral entropy showed a pretty limited variability, large delta variation occurred along with discontinuous signs of sleep from about 22:30 to 4:30) and again between 7:00 and 9:30. The $24 \mathrm{~h}$ mean amplitude spectrum highlighted the presence of a periodicity of $9 \mathrm{~h}$. When considering the period lasting from 9:30 to $17: 30$ no main periodicity could be found (Fig. J of Supplementary Material).

\section{Discussion}

We here analyzed $4 \mathrm{~h}$ EEG recordings in 12 patients with disorders of consciousness. For the sake of robustness we will discuss only those results which were proven significant for all the three considered EEG derivations $(\mathrm{Fz}, \mathrm{Cz}$ and $\mathrm{Pz}$ ). As expected, based on findings from previous studies, the MCS group $(n=6)$ showed higher relative power in theta and alpha bands whereas the VS/UWS group showed a higher relative power within the delta band. These results corroborate the findings from Sitt et al. [22], who observed a monotonically decreasing power in the EEG delta range when moving from VS/UWS to full consciousness. These authors also reported that relative theta and alpha powers discriminated MCS from VS/UWS. Similarly, Lehembre et al. [23], demonstrated that VS/ UWS patients showed higher delta and lower alpha activity as compared to MCS. In addition to their findings, we here observed also a higher relative power within theta band in MCS patients. Several other studies reported that theta activity is prominent in MCS, both in case of widespread cortical damage [24] and of focal brain lesions [25]. Next, we observed higher EEG spectral entropy values in MCS as compared to VS/UWS, confirming findings described in a previous study from Gosseries et al. [16].

While the above-discussed results represent a confirmation of findings already described in literature, the originality of the present study stems from the characterization of the temporal dynamics of EEG spectral entropy changes in disorder of consciousness patients. Our data show that spectral entropy time variability is higher in MCS as compared to VS/UWS. It could be argued that the higher spectral entropy time variability could simply be a consequence of higher mean spectral entropy in MCS. In order to rule out this interpretation, we took into account not only the standard deviation across time but also the coefficient of variation which was still significantly higher in MCS as compared to VS/UWS. This finding was confirmed by results of correlation analyses between total CRS-R scores and spectral entropy variability features (12 patients), showing that CRS-R scores were positively correlated to spectral entropy variability $(r=0.857$, $p<0.006$ for the standard deviation and $r=0.848$, $p<0.006$ for the coefficient of variation).

In our view, these results could reflect the variability of behavioral responses characteristic of MCS, [2, 4]. Indeed, their level of consciousness is known to undergo large fluctuations ranging from time lapses of total unresponsiveness to moments in which a certain cognitively mediated behavior can be detected. We suggest that the EEG spectral entropy variability in MCS could mirror the fluctuation of awareness described in this cohort of patients.

As a further step, we verified whether the spectral entropy time-variability of the patients had a specific ultradian periodicity or its fluctuations occurred in a random fashion. Spectral entropy time-courses were therefore submitted to a time-frequency wavelet analysis. Patients in VS/UWS showed inconsistent periodicities (25-120 min) whereas all MCS patients included in the studied 
convenience sample showed well-defined spectral entropy cyclicities (ranging from 53 to $80 \mathrm{~min}$ ) with a mean periodicity peak at $70 \mathrm{~min}$. These findings are in agreement with Cruse et al. [26], who previously showed that VS/ UWS patients had greater impairment of circadian motor rhythmicity when compared to MCS when analyzing multiple day recordings of wrist-actigraphy.

Spectral entropy periodicity could be strongly affected by vigilance fluctuations, be they in the wakefulness spectrum (mental rest/increased mental activity [27]), or even including shifts towards drowsiness or NREM sleep. The latter events in particular, if proven true, could strongly bias the interpretation of the novel findings herein described, especially when considering the MCS group. Periods of lower vigilance are usually characterized by reduced muscular and ocular activity, accompanied by a slowing of EEG patterns, which at least when considering healthy subjects, results in an enhancement of theta activity during drowsiness and in the appearance of delta waves during NREM sleep. It is worth underlining that states of extremely low vigilance are not always characterized by the presence of high amplitude theta/and or delta activity, even when considering healthy subject: as an example REM sleep is a condition of low vigilance and relatively high awareness [28], characterized by low voltage theta and beta activities. Conversely, MCS and VS/UWS patients can still reach vigilance levels comparable to those of conscious wakefulness [28], while being characterized at the EEG level respectively by theta (at least for a sub-group of MCS $[24,25])$ and delta activity patterns.

The presence of vigilance shifts during the $4 \mathrm{~h}$ of recording, and their putative influence on spectral entropy fluctuations was verified for each group by comparing their spectral entropy periodicity to those of EOG, EMG and EEG bands power time-series. None of the considered features showed a significant main periodicity comparable to that of the spectral entropy, with the notable exception of beta2 band in the MCS group. When considering delta band, we found analogous periodicity distributions when comparing MCS to VS/UWS: both distributions had a maximum at $120 \mathrm{~min}$ and a local peak of lower amplitude at $60 \mathrm{~min}$ (see Fig. 6). As a further check, the correlation between spectral entropy time-course and EOG, EMG and EEG-band powers time-courses was estimated for each subject. No significant correlation was found for any of the subjects either for EOG or EMG activity (only one VS/ UWS patient showed a significant anti-correlation with the ocular activity), while in case of conspicuous vigilance fluctuation we would have expected significant anti-correlations between EOG and EMG activities on the one side and spectral entropy (i.e. higher vigilance states are characterized by higher ocular and muscular activities).
A heterogeneous set of significant correlations (either positive or negative), were found when considering the time-courses of power in the EEG bands of interest, but no coherent pattern of correlations could be found even at the single group level. On the basis of these findings, three main conclusions can be drawn:

- During the considered $4 \mathrm{~h}$ subjects did not exhibit any relevant drop of vigilance.

- No evidence of a significant influence of vigilance shifts on spectral entropy fluctuations could be found.

- Spectral entropy fluctuations are possibly driven by the interplay of different frequency bands' activities; the involved bands are patient-specific, depending (by inference) on the level of preservation of his/her cortical and sub-cortical structures and on brain residual connectivity.

To the best of our knowledge, no previous study has investigated the presence of ultradian rhythms in disorders of consciousness during wakefulness. Tsuji and Kobayashi [27] found that diurnal EEG rhythms of healthy subjects are composed by two different, yet superimposable, ultradian components: (1) a short periodicity ranging from 70 to $110 \mathrm{~min}$, and (2) a long periodicity ranging from 3 to $8 \mathrm{~h}$. The faster rhythm was hypothesized to be related to fluctuations in vigilance/global attention (i.e., an oscillation between a state of mental rest and one of increased mental activity). This rhythmicity could possibly reflect the basic rest-activity cycle initially proposed by Kleitman [29, 30]. Similar results were described also by Okawa et al. [31], who detected the presence of vigilance fluctuations during daytime with periods ranging between 60 and $110 \mathrm{~min}$ and by Manseau and Broughton [32] who documented the presence (in normal volunteers) of ultradian variations of the EEG power spectra in the $4-20 \mathrm{~Hz}$ band with cyclicities between 72 and 120 min: these cyclicities are similar to the those we observed in severely brain-damaged minimally conscious patients who (on average) showed a $70 \mathrm{~min}$ periodicity. The authors suggested that this ultradian rhythmicity could be linked to brainstem and diencephalic ascending activating reticular formation, known to modulate vigilance/global attention [33,34]. The cycles' lengths identified in our MCS group (53-80 min) are slightly lower than the $70-120$ min periodicity previously shown in healthy subjects. This might be caused by a dysfunction of brainstem and/or central thalamic structures, known to be structurally damaged in MCS [35]. In this framework we found that higher total CRS-R scores (mirroring higher levels of awareness/vigilance, at least in our cohort of patients, see Fig. E, Supplementary Material) are paralleled in the MCS group by longer SE periodicities $(r=0.937$, $p<0.02$ ), closer to those typical of healthy subjects. 
Of note, two VS/UWS patients showed local periodicities ( $\mathrm{Cz}$ and/or Pz electrodes) compatible with those found in the MCS group. This finding is potentially interesting as it could be related to a partial anatomo-functional preservation of the structures underlying the electrodes and conversely the absence of such periodicities on Fz could reflect severe damages of the underlying cortical structures. However such findings might be biased by volume conduction effects and should thus be further tested using dense-array EEG systems and possibly electrical source imaging techniques. Apart from the two above-mentioned exceptions, we here failed to identify a reliable ultradian rhythmicity in EEG spectral entropy in the VS/UWS patients group. This could be either due to the relatively low length of the EEG recordings that did not allow for the identification of putative slower ultradian rhythmicity (i.e., with periodicity higher than $120 \mathrm{~min}$ ) or to the absence of any periodical pattern in these patients. Another possible factor influencing the absence of consistent periodical patterns in the VS/UWS group could have been the inclusion of three sub-acute patients (time since insult 15-16 days). We ruled out this potential bias, at least for our cohort of VS/UWS patients, confronting their normalized powers, spectral entropy features, and periodicities (see Fig. F-H and Tables G, H, Supplementary Material). The comparison of the EEG features did not reveal any systematic or relevant difference between the sub-acute and chronic sub-group. It is still fair to underline that the absence of relevant EEG differences between sub-acute and chronic patients which holds in our selected sample, it is hardly generalizable given the small sample size, but could rather be a hypothesis to be tested on larger cohorts of VS/UWS. More in general the results of this preliminary study based on a relatively small cohort of patients should be confirmed by further studies on larger samples of patients.

Longer recording times will thus be needed to verify the presence or absence of slower ultradian rhythms in disorders of consciousness patients, taking into account the possible influence of etiology and duration since the onset of the disorder as including in the same study chronic and sub-acute patients could introduce an unwanted confounding factor. Ideally, long-duration EEG spectral analyses should also be confronted with repeated behavioral measures of vigilance and awareness but also with structural and functional neuroimaging data (i.e., MRI and PET), permitting to characterize the functional neuro-anatomy of ultradian rhythmicity alterations after coma and their clinical impact on the patient's diagnosis and prognosis.

As a proof-of-concept of the feasibility of spectral entropy fluctuation analysis on $24 \mathrm{~h}$ recordings, we report the results pertaining two exemplary patients (MCS3 and VS/UWS5). For MCS3 an ultradian periodicity of $7 \mathrm{~h}$ was found (possibly related to sleep cycles, as at least two cycles were detected in the period starting at 22:30 and ending at about 6:00). Moreover, when considering the period lasting from 9:30 to 17:30, the presence of the same 65 min periodicity found in the first $4 \mathrm{~h}$ of recording was verified: this periodicity could thus be considered as a stable constitutive feature of the patient's brain activity during wakefulness.

Regarding the VS/UWS subject, discontinuous signs of sleep were detected from about 22:30 to 4:30 and again between 7:00 and 9:30. The $24 \mathrm{~h}$ mean amplitude spectrum highlighted the presence of a $9 \mathrm{~h}$ periodicity whereas no main periodicity was found in the eyes-open period (9:30$17: 30)$. It is worth underlining that this latter subject, beyond the difficulties inherent to the EEG analysis common to all VS/UWS patients (especially when dealing with sleep detection), was even more challenging given the progressive worsening of the EEG signal quality, which severely undermines the reliability of the presented results.

We have here identified the existence of an EEG ultradian rhythm with a cyclicity of about 70 min seemingly characteristic of the spectral entropy time-course of patients in MCS. We interpret this cyclicity as a possible electrophysiological index reflecting the vigilance/awareness fluctuations encountered after severe brain damage. If our hypothesis would be confirmed, spectral entropy monitoring could be considered to all clinical and scientific intents and purposes as a marker of consciousness level fluctuations. Automated real-time EEG entropy measures could allow clinicians and scientists to choose specific appropriate time-windows for performing bedside behavioral assessments of consciousness and complementary electrophysiological (ERP) or functional neuroimaging tests thus overriding the inconsistencies in cognitively mediated behaviors and possibly lowering the rate of misdiagnosis. Related to electrophysiological and functional neuroimaging, both "resting state", passive and active activation paradigms in EEG, MEG or imaging studies could benefit from continuous EEG spectral entropy monitoring. Lastly, the identification of periods of higher/ lower awareness could be of help in optimizing the timing of delivery when using therapeutic techniques such as physical therapy and electrical brain stimulation (e.g., transcranial direct current stimulation [36]).

Acknowledgments No external funding was used for the study.

\section{Compliance with ethical standards}

Conflicts of interest None of the authors have potential conflicts of interest to be disclosed.

Ethical standards The study was approved by the Ethics Committee of the Faculty of Medicine of the University of Liège. Written informed consent forms were received from the patients' legal representatives. The study was conducted in agreement with the Declaration of Helsinki 1964 and its later amendments. 


\section{References}

1. Laureys S, Celesia GG, Cohadon F, Lavrijsen J, León-Carrión J, Sannita WG, Sazbon L, Schmutzhard E, von Wild KR, Zeman A, Dolce G, Force European Task, on Disorders of Consciousness (2010) Unresponsive wakefulness syndrome: a new name for the vegetative state or apallic syndrome. BMC Med 8:68

2. Giacino JT, Ashwal S, Childs N, Cranford R, Jennett B, Katz DI, Kelly JP, Rosenberg JH, Whyte J, Zafonte RD, Zasler ND (2002) The minimally conscious state, definition and diagnostic criteria. Neurology 58:349-353

3. Bruno MA, Vanhaudenhuyse A, Thibaut A, Moonen G, Laureys S (2011) From unresponsive wakefulness to minimally conscious PLUS and functional locked-in syndromes: recent advances in our understanding of disorders of consciousness. J Neurol 258:1373-1384

4. Majerus S, Bruno MA, Schnakers C, Giacino JT, Laureys S (2009) The problem of aphasia in the assessment of consciousness in brain-damaged patients. Prog Brain Res 177:49-61

5. Giacino JT, Fins JJ, Laureys S, Schiff ND (2014) Disorders of consciousness after acquired brain injury: the state of the science. Nat Rev Neurol 10:99-114

6. Kotchoubey B, Vogel D, Lang S, Müller F (2014) What kind of consciousness is minimal? Brain Inj 28:1156-1163

7. Monti MM, Laureys S, Owen AM (2010) The vegetative state. BMJ 341:c3765

8. Giacino JT, Kalmar K, Whyte J (2004) The JFK Coma Recovery Scale-Revised: measurement characteristics and diagnostic utility. Arch Phys Med Rehabil 85:2020-2029

9. Seel RT, Sherer M, Whyte J, Katz DI, Giacino JT, Rosenbaum AM, Hammond FM, Kalmar K, Pape TL, Zafonte R, Biester RC, Kaelin D, Kean J, Zasler N (2010) Assessment scales for disorders of consciousness: evidence-based recommendations for clinical practice and research. Arch Phys Med Rehab 91:1795-1813

10. Monti MM, Vanhaudenhuyse A, Coleman MR, Boly M, Pickard JD, Tshibanda L, Owen AM, Laureys S (2010) Willful modulation of brain activity in disorders of consciousness. N Engl J Med 362:579-589

11. Laureys S, Schiff ND (2012) Coma and consciousness: paradigms (re)framed by neuroimaging. Neuroimage 61:478-491

12. Gosseries O, Zasler ND, Laureys S (2014) Recent advances in disorders of consciousness: focus on the diagnosis. Brain Inj 28:1141-1150

13. Gosseries O, Di H, Laureys S, Boly M (2014) Measuring consciousness in severely damaged brains. Annu Rev Neurosci 37:457-478

14. Mahon P, Greene BR, Lynch EM, McNamara B, Shorten GD (2008) Can state or response entropy be used as a measure of sleep depth? Anaesthesia 63:1309-1313

15. Vanluchene AL, Vereecke H, Thas O, Mortier EP, Shafer SL, Struys MM (2004) Spectral entropy as an electroencephalographic measure of anesthetic drug effect: a comparison with bispectral index and processed midlatency auditory evoked response. Anesthesiology 101:34-42

16. Gosseries O, Schnakers C, Ledoux D, Vanhaudenhuyse A, Bruno MA, Demertzi A, Noirhomme Q, Lehembre R, Damas P, Goldman S, Peeters E, Moonen G, Laureys S (2011) Automated EEG entropy measurements in coma, vegetative state/unresponsive wakefulness syndrome and minimally conscious state. Funct Neurol 26:25-30

17. Cologan V, Drouot X, Parapatics S, Delorme A, Gruber G, Moonen G, Laureys S (2013) Sleep in the unresponsive wakefulness syndrome and minimally conscious state. J Neurotrauma 30:339-346

18. Manly BJF (2006) Randomization, bootstrap and Monte Carlo methods in biology, 3rd edn. Chapman \& Hall/CRC, London

19. Benjamini Y, Yekutieli D (2001) The control of the false discovery rate in multiple testing under dependency. Ann Statist 29:1165-1188

20. Torrence C, Compo GP (1998) A practical guide to wavelet analysis. B Am Meteorol Soc 79:61-78

21. Shapiro SS, Wilk MB (1965) An analysis of variance test for normality (complete samples). Biometrika 52:591-611

22. Sitt JD, King JR, El Karoui I, Rohaut B, Faugeras F, Gramfort A, Cohen L, Sigman M, Dehaene S, Naccache L (2014) Large scale screening of neural signatures of consciousness in patients in a vegetative or minimally conscious state. Brain 137:2258-2270

23. Lehembre R, Bruno MA, Vanhaudenehuyse A, Chatelle C, Cologan V, Leclerq Y, Soddu A, Macq B, Laureys S, Noirhomme Q (2012) Resting-state EEG study of comatose patients: a connectivity and frequency analysis to find differences between vegetative and minimally conscious states. Funct Neurol 27:41-47

24. Williams ST, Conte MM, Goldfine AM, Noirhomme Q, Gosseries Q, Thonnard M, Beattie B, Hersh J, Katz DI, Victor JD, Laureys S, Schiff ND (2013) Common resting brain dynamics indicate a possible mechanism underlying zolpidem response in severe brain injury. ELife 2:e01157

25. Carboncini MC, Piarulli A, Virgillito A, Arrighi PA, Andre P, Tomaiuolo F, Frisoli A, Bergamasco M, Rossi B, Bonfiglio L (2014) A case of post-traumatic minimally conscious state reversed by midazolam: clinical aspects and neurophysiological correlates. Restor Neurol Neuros 32:767-787

26. Cruse D, Thibaut A, Demertzi A, Nantes JC, Bruno MA, Gosseries O, Vanhaudenhuyse A, Bekinschtein TA, Owen AM, Laureys S (2013) Actigraphy assessments of circadian sleepwake cycles in the vegetative and minimally conscious states. BMC Med 11:18

27. Tsuji Y, Kobayashi T (1988) Short and long ultradian EEG components in daytime arousal. Electroen Clin Neuro 70:110-117

28. Laureys S (2007) Eyes open, brain shut. Sci Am 296:84-89

29. Kleitman N (1961) The nature of dreaming. In: Wolstenholme GEW, O'Connor M (eds) The nature of sleep. Churchill, London, pp 349-364

30. Kleitman N (1982) Basic rest-activity cycle-22 years later. Sleep 5:311-317

31. Okawa M, Matousek M, Petersén I (1984) Spontaneous vigilance fluctuations in the daytime. Psychophysiology 21:207-211

32. Manseau C, Broughton RJ (1984) Bilaterally synchronous ultradian EEG rhythms in awake adult humans. Psychophysiology $21: 265-273$

33. Kinomura S, Larsson J, Gulyás B, Roland PE (1996) Activation by attention of the human reticular formation and thalamic intralaminar nuclei. Science 271:512-515

34. Saper CB, Scammell TE, Lu J (2005) Hypothalamic regulation of sleep and circadian rhythms. Nature 437:1257-1263

35. Schiff ND (2008) Central thalamic contributions to arousal regulation and neurological disorders of consciousness. Ann NY Acad Sci 1129:105-118

36. Thibaut A, Bruno MA, Ledoux D, Demertzi A, Laureys S (2014) tDCS in patients with disorders of consciousness: sham-controlled randomized double-blind study. Neurology 82:1112-1118 